\title{
Updates in Cartilage Tissue Regeneration
}

\author{
Gun-Il $\mathrm{Im}^{1} \cdot$ Chong-Su Cho ${ }^{2}$
}

Published online: 30 July 2019

(C) The Korean Tissue Engineering and Regenerative Medicine Society 2019

It has long been known that articular cartilage does not regenerate itself. Because unhealed chondral defects ultimately lead to osteoarthritis, a lot of surgical or nonsurgical methods have been developed and applied to patients without noticeable success. Cell therapy for regeneration of articular cartilage defects was firstly tried using autologous chondrocytes in 1994. Tissue engineering-based approaches that employ guiding biomaterials and bioactive agents in addition to cell implantation have been also applied for the regeneration of articular cartilage. Unlike the regeneration of bone, the attempts for cartilage tissue regeneration did not uniformly lead to great clinical success so far. Various concepts and strategies have appeared and vanished in last 25 years [1-3].

In this special issue, updates on recent thoughts and techniques on cartilage tissue regeneration are introduced. Van der Kraan discussed on the interaction between joint inflammation and cartilage repair. He stressed that the relationship between inflammation and tissue repair was not straightforward. While acute, local inflammation is deleterious for chondrogenesis and cartilage repair, systemic inflammation exerts a negative effect on all kinds of tissue repair. Importantly, as the currently widely used models of chondrogenesis employ high differentiation factor concentrations and corticosteroid levels that

Gun-Il Im

gunil@duih.org

1 Integrative Research Institute for Regenerative Medical Engineering, Dongguk University, 27 Dongguk-ro,

Ilsandong-gu, Goyang-si, Gyeonggi-do 10326, Republic of Korea

2 Research Institute of Agriculture and Life Sciences, Seoul National University, 1 Gwanak-ro, Gwanak-gu, Seoul 08826, Republic of Korea suppress inflammation, model systems must be developed that are better to mimic the real conditions in a joint with damaged cartilage [4]. Choi et al. summarized the current state of knowledge in genetic and epigenetic associations and risk factors for osteoarthritis and application for diagnostic and therapeutic purpose. The studies confirmed that there was a genetic basis for certain subsets of osteoarthritis pathogenesis. They expected that genome editing technologies such as the CRISPR-Cas9 system could be used as platforms from which potential biomarkers for the diagnosis, prognosis, drug response, and development of potential personalized therapeutic targets for osteoarthritis could be approached. Also, the development of "designer" cells, whereby the receptors, gene regulatory networks, or transgenes can be modified as a basis for new cell-based therapies, will be made possible with genome editing technologies [5]. Venkatesan et al. provided an overview on exploiting biomaterial technologies and therapeutic viral gene transfer for musculoskeletal regeneration. They highlighted that combining viral gene therapy techniques with tissue engineering procedures might offer strong tools to improve the current systems for applications in vivo and overcome the obstacles precluding effective viral gene therapy. Biomaterial-guided viral gene therapy has a strong potential for translation in the field of musculoskeletal regenerative medicine [6]. Im summarized current progress of IA injection therapy and the author's perspective on the IA therapy including cell injection. He concluded that it is too premature to have any conclusion on the eventual efficacy of IA cell therapy concerning regeneration of articular cartilage-based current data because prospective radiological and histological data from larger numbers of patients are needed to prove cost effectiveness of IA cell therapy. He expected that expanding researches in this field would produce further evidences to 
provide guidance on the eventual effectiveness of IA cell therapy in the future [7].

This special issue provides perspectives on the concept and techniques on the cartilage tissue regeneration that may be employed for patients care in the future. We hope this special issue will help to advance and benefit human health and well-being.

Acknowledgement The editors express a deep sense of gratitude to all the invited authors for their valuable efforts for the review articles.

\section{References}

1. Im GI. Gene transfer strategies to promote chondrogenesis and cartilage regeneration. Tissue Eng Part B Rev. 2016;22:136-48.

2. Im GI. Clinical use of stem cells in orthopaedics. Eur Cell Mater. 2017;33:183-96.
3. Ko JY, Lee J, Lee J, Im GI. Intra-articular xenotransplantation of adipose-derived stromal cells to treat osteoarthritis in a goat model. Tissue Eng Regen Med. 2017;14:65-71.

4. van der Kraan PM. The interaction between joint inflammation and cartilage repair. Tissue Eng Regen Med. 2019. https://doi.org/10. 1007/s13770-019-00204-z.

5. Choi YR, Collins KH, Lee JW, Kang HJ, Guilak F. Genome engineering for osteoarthritis: from designer cells to diseasemodifying drugs. Tissue Eng Regen Med. 2019. https://doi.org/10. 1007/s13770-018-0172-4.

6. Venkatesan JK, Rey-Rico A, Cucchiarini M. Current trends in viral gene therapy for human orthopaedic regenerative medicine. Tissue Eng Regen Med. 2019. https://doi.org/10.1007/s13770-01900179-x.

7. Im GI. Perspective on intra-articular injection cell therapy for osteoarthritis treatment. Tissue Eng Regen Med. 2019. https://doi. org/10.1007/s13770-018-00176-6.

Publisher's Note Springer Nature remains neutral with regard to jurisdictional claims in published maps and institutional affiliations. 